\title{
Alimentary satisfaction, gastrointestinal symptoms, and quality of life 10 or more years after esophagectomy with gastric pull-up
}

\author{
Christina L. Greene, MD, Steven R. DeMeester, MD, Stephanie G. Worrell, MD, Daniel S. Oh, MD, \\ Jeffrey A. Hagen, MD, and Tom R. DeMeester, MD
}

Objective: The aim of this study was to evaluate alimentary satisfaction, gastrointestinal symptoms, and quality of life $\geq 10$ years after esophagectomy with gastric pull-up.

\begin{abstract}
Methods: Patients who had undergone esophagectomy with gastric pull-up before 2003 were interviewed regarding their alimentary function and completed the Gastrointestinal Quality of Life and RAND short-form, 36-item, questionnaires.

Results: We identified 67 long-term survivors after esophagectomy and gastric pull-up. Of these, 40 were located, and all agreed to participate. The median age was 75 years, and the median follow-up period was 12 years (interquartile range, 10-19). Most patients (88\%) had no dysphagia, $90 \%$ were able to eat $\geq 3$ meals/ day, and $93 \%$ finished $\geq 50 \%$ of a typical meal. The mean alimentary comfort rating was 9 of 10 . Dumping, diarrhea $\geq 3$ times/day, or regurgitation occurred in $33 \%$ of patients. Six patients $(15 \%)$ had aspiration episodes requiring hospitalization. The median weight loss after surgery was $26 \mathrm{lbs}$, and the current median body mass index was $25 \mathrm{~kg} / \mathrm{m}^{2}$. Only 2 patients were underweight (body mass index, $<18.5 \mathrm{~kg} / \mathrm{m}^{2}$ ). The median Gastrointestinal Quality of Life score was 2.9 of 4 . The RAND scores were at the population mean in 1 category (physical function) and above the normal mean in the remaining 7 categories.
\end{abstract}

Conclusions: Long-term nutritional status, quality of life, and satisfaction with eating were excellent after esophagectomy with gastric pull-up. Gastrointestinal side effects were common, but serious complications such as aspiration were uncommon. Pessimism regarding the long-term ability to enjoy a meal and live with a good quality of life after esophagectomy is unwarranted. (J Thorac Cardiovasc Surg 2014;147:909-14)

Esophageal cancer is a lethal disease, which often leads to pessimism on the part of treating physicians and patients. ${ }^{1}$ Esophagectomy with gastric pull-up, either alone for early disease or after neoadjuvant therapy for patients with more extensive locoregional disease, is potentially curative. However, the morbidity of the procedure and the uncertainty about postesophagectomy quality of life has led patients to consider alternative options. Esophagectomy has been shown to have a significant negative effect on physical fitness and health-related quality of life (HRQL), with only minor recovery 6 months to 3 years after the procedure. ${ }^{2-6}$ Furthermore, dysphagia, anastomotic strictures, dumping, and diarrhea have been common early after esophagectomy. ${ }^{7}$ Little is known, however, about the

From the University of Southern California Keck School of Medicine, Los Angeles, Calif.

Disclosures: Steven R. DeMeester reports consulting fees for Bard, C2 Therapeutics, and Novadaq, as well as lecture fees from Bard. All other authors have nothing to disclose with regard to commercial support.

Read at the 39th Annual Meeting of The Western Thoracic Surgical Association, Coeur d'Alene, Idaho, June 26-29, 2013.

Received for publication June 26, 2013; revisions received Oct 28, 2013; accepted for publication Nov 7, 2013; available ahead of print Dec 11, 2013.

Address for reprints: Steven R. DeMeester, MD, University of Southern California Keck School of Medicine, 1510 San Pablo St, Suite 514, Los Angeles, CA 90033 (E-mail: Steven.DeMeester@med.usc.edu).

$0022-5223 / \$ 36.00$

Copyright (c) 2014 by The American Association for Thoracic Surgery

http://dx.doi.org/10.1016/j.jtcvs.2013.11.004 recovery of gastrointestinal function and long-term quality of life after esophagectomy. The aim of the present study was to evaluate alimentary satisfaction, gastrointestinal symptoms, and quality of life $\geq 10$ years after esophagectomy with gastric pull-up.

\section{METHODS}

\section{Patients}

A retrospective chart review was performed to identify all patients who had undergone esophagectomy with gastric pull-up from 1993 to 2002 . The Social Security Death Index website was queried to identify the surviving patients. An exhaustive search was performed to locate the patients' current contact information. The patients were contacted by telephone and interviewed regarding their alimentary satisfaction and reflux symptoms. They were also asked to complete the Gastrointestinal Quality of Life Index (GIQLI) and RAND Medical Outcomes Study short-form, 36-item (SF-36) questionnaire. The date and results of their last endoscopy (if not done at our center) were requested. The demographic and operative information, symptoms (past and present), acid suppression medication usage, endoscopic findings, and quality of life information were recorded in a database. The institutional review board of the University of Southern California approved this study.

\section{Assessment of Alimentary Satisfaction and Gastrointestinal Symptoms}

Telephone interviews were conducted by a single investigator using a standard format (Table 1). The patients were asked to rate their overall alimentary tract comfort on a scale from 0 to 10 . A score of 0 indicated that their current alimentary function was intolerable, and a score of 10 indicated that they were completely satisfied and could not ask for anything better. 


\section{Abbreviations and Acronyms \\ GIQLI = Gastrointestinal Quality of Life Index \\ $\mathrm{HRQL}=$ health-related quality of life \\ $\mathrm{IQR}=$ interquartile range \\ SF-36 = RAND Medical Outcomes Study short- form, 36-item}

\section{Assessment of Quality of Life}

The GIQLI is a measure of the quality of life for patients with gastrointestinal disease. ${ }^{8}$ It is a validated 36 -item survey. Each question is scored from 0 to 4 , with higher scores denoting better function. The survey includes 5 domains of questions (core symptoms, physical dysfunction, psychological dysfunction, social dysfunction, and diseasespecific dysfunction). The score for each domain is the patient's average score for those questions. The overall score ranges from 0 to 144 .

The SF-36 was designed to characterize a person's view of their health and quality of life. ${ }^{9}$ It evaluates 8 areas, including physical functioning, bodily pain, limitations due to physical health problems, limitations due to personal or emotional problems, emotional well-being, social functioning, energy/fatigue, and general health perceptions. This version has been adapted from the Medical Outcome Study and is published by RAND. Items are scored on a scale from 0 to 100 , with higher scores denoting better function. The scores in the same areas are then averaged to create 8 scale scores.

Every effort was made to obtain complete information for each question. When the questionnaires were incomplete, the patients were interviewed again in an attempt to acquire the missing responses. The scores from the GIQLI and RAND SF-36 were compared with published normal US population values. ${ }^{8-10}$

\section{Statistical Analysis \\ The data were analyzed using the mean and median values. The median values are reported with the interquartile range (IQR), and the mean values are reported with the range and/or standard deviation. The Pearson product-moment correlation coefficient was calculated to analyze the temporal relationship between the postoperative duration, symptomatic outcome, and quality of life. In the event that the information for a question was unavailable, the missing responses were omitted, and the weighting for that questionnaire was adjusted.}

\section{RESULTS}

A total of 398 patients had undergone esophagectomy with gastric pull-up during the study period. Of these, 67 were confirmed to be alive, and 40 were located (36 men and 4 women). All 40 agreed to participate. The median age at esophagectomy was 61 years (IQR, 48-81), and the current median age at the follow-up point was 75 years (IQR, 58-92). The indication for esophagectomy was cancer in 39 patients and benign disease in 1 . Of the 40 patients, 8 had undergone neoadjuvant chemotherapy without radiotherapy and 3 patients had undergone adjuvant chemotherapy. The final pathologic stage for the 40 patients is listed in Table 2. The esophagectomy approach was transthoracic en bloc in 24 and transhiatal in 16 patients. In all patients, the graft was positioned in the posterior mediastinum, and a cervical esophagogastrostomy constructed. A pyloroplasty was performed in all but 1 patient, who underwent vagal-sparing esophagectomy.

The median follow-up period was 12 years (IQR, 10-19). Most patients (88\%) had no dysphagia, $90 \%$ were able to eat $\geq 3$ meals/day, and $93 \%$ could finish $\geq 50 \%$ of a typical meal (Figure 1). The mean rating of alimentary comfort was 9 on a 10-point scale (range, 5.5-10); $38 \%$ were completely satisfied with a score of 10 of 10 , and 2 rated their satisfaction at 5.5 (Table 3). All patients had made dietary modifications to avoid problematic foods. The most commonly avoided food was sweet dairy products such as ice cream and milkshakes. The most common gastrointestinal symptoms were early satiety $(50 \%)$, dumping $(33 \%)$, diarrhea $>3$ times/day (30\%), and regurgitation (30\%). Chronic cough was present in $10 \%$, and $25 \%$ had intermittent episodes of dyspnea. Detailed information on dumping symptoms and acid suppression medication use was available from 31 patients. The frequency of dumping is shown in Figure 2. Acid suppression medications, most commonly a proton pump inhibitor, were used by $74 \%$ of the patients.

All but 1 patient slept on a wedge pillow or hospital bed. Most patients, on occasion, had an episode of nocturnal aspiration after they had slid down or rolled off their wedge pillow. These events had often been followed by 48 hours of flu-like symptoms. Aspiration requiring hospitalization occurred in 6 patients (15\%), and all had $\geq 3$ hospitalizations in the past year alone. At least 1 dilatation for an anastomotic stricture was necessary in 12 patients $(30 \%)$, but no patient required dilatation at the follow-up point. The median postoperative weight loss was $26 \mathrm{lbs}$. The median body mass index (BMI) at the time of surgery was $28 \mathrm{~kg}$ / $\mathrm{m}^{2}$, and the median BMI at follow-up was $25 \mathrm{~kg} / \mathrm{m}^{2}$. All but 2 patients weighed less than their preoperative weight, but only 2 patients were underweight by World Health Organization standards (BMI, $<18.5 \mathrm{~kg} / \mathrm{m}^{2}$ ). The percentage of patients who were overweight decreased after surgery, with $54 \%$ of patients in the normal BMI range compared with $14 \%$ preoperatively. The BMI distribution is listed in Table 4. No patient required supplemental tube feeding or was using nutritional supplementation to maintain their weight at the follow-up point.

The median and mean cumulative GIQLI score for all domains was 104 (IQR, 76.5-120) and $88.7 \pm 41.1$, respectively. The GIQLI subdomain scores are shown in Figure 3. The highest median scores were for social functioning (3.8) and gastrointestinal disease-specific symptoms (3.3), and the lowest score was for physical functioning (1.9).

The SF-36 quality of life scores were at the population mean for 1 category (physical function) and were greater than the mean for the remaining 7 (role limitation due to physical health, role limitation due to emotional health, energy/fatigue, emotional well-being, social functioning, pain, and general health; Figure 4). No relationship was 
TABLE 1. Telephone interview questions

Have you had any hospital admission or surgeries since your esophagectomy?

Do you have difficulty swallowing?

Do you have painful swallowing or a sticking sensation in your throat when you eat?

How many meals do you eat per day?

Are you able to eat $\geq 50 \%$ your meal?

Are you always the last to finish eating? Do your friends and family have to wait for you because it takes you so long to eat?

Do you have heart palpitations when you eat?

Do you have sweating when you eat?

Do you get full easily when you eat?

Do you have $\geq 3$ episodes of diarrhea a day?

Do you have regurgitation? Do you feel it to be disabling?

Have you ever woken up in the middle of the night choking or been admitted/treated for aspiration?

Do you have heartburn and do you think it is disabling?

What medications are you currently taking?

Do you experience shortness of breath? Have you developed COPD?

Do you smoke or have you ever smoked and for how long?

Do you have a chronic cough?

Have you noticed any trends over time that I have not addressed?

What was the date and location of your last endoscopy? Can you please send us a copy?

On a scale of $0-10$, with 10 indicating you are very comfortable and 0 , you are very uncomfortable, how would you rate your overall comfort with your alimentary tract as it currently stands, including chewing,

swallowing, and digesting everything.

$C O P D$, Chronic obstructive pulmonary disease.

seen between the postoperative duration and outcomes, including the quality of life, when analyzed using Pearson correlation coefficients (data not shown). The subgroup analysis showed no difference in the quality of life scores between the patients who had received neoadjuvant therapy versus primary surgery or transhiatal esophagectomy versus en bloc esophagectomy.

\section{DISCUSSION}

Esophagectomy with gastric pull-up is a major physiologic insult to the entire body and also produces significant gastrointestinal alterations. Most commonly, it has been

TABLE 2. Pathologic stage $(n=40)$

\begin{tabular}{lc}
\hline Final pathologic stage & Patients \\
\hline Stage IA & $22(55)$ \\
Stage IB & $2(5)$ \\
Stage IIA & $1(2.5)$ \\
Stage IIB & $4(10)$ \\
Stage IIIA & $3(7.5)$ \\
yP0 & $3(7.5)$ \\
yPIA & $1(2.5)$ \\
yPIIA & $2(5)$ \\
yPIIIA & $2(5)$ \\
\hline
\end{tabular}

Data presented as n $(\%)$. done as part of a curative strategy for cancer. However, on occasion, it has been performed in an effort to improve alimentation in patients with end-stage benign disease. In the first 6 months after esophagectomy, it has been shown that fatigue, dyspnea, and diarrhea were all worse compared with the pre-esophagectomy baseline; however, for patients with cancer, their emotional function had significantly improved. ${ }^{6}$ Studies evaluating the effect of major postoperative complications found that although they do not affect the global quality of life, they were associated with significantly more eating restrictions and problems with choking, fatigue, and dyspnea. ${ }^{6}$ The problems tended to improve with time, but the differences persisted even out to 5 years. The longest reported follow-up for quality of life after esophagectomy has been 5 years, with several studies reporting that the HRQL, as a whole, was comparable to the national norms, although physical functioning and reflux remained problematic. ${ }^{12}$ The extent of lymphadenectomy and the approach for esophagectomy did not appear to affect the HRQL measures. However, elective esophagectomy in patients with cancer led to more pronounced deficits in physical functioning than that reported by patients who had undergone urgent or emergent esophagectomy for a benign process. ${ }^{13,14}$ These findings suggest that some of the physical function impairments after esophagectomy for cancer result from the cancer itself and the associated treatment with chemotherapy and/or radiotherapy. In our small group of long-term survivors after esophagectomy and gastric pull-up, we found no difference in physical function impairments between the 8 patients who had undergone neoadjuvant chemotherapy and those treated with primary esophagectomy. No patient in the present series had undergone radiotherapy; thus, we were unable to assess for any long-term implications of radiotherapy for esophageal cancer. In addition, only 1 patient had undergone esophagectomy for benign disease in our group. Consequently, we were unable to assess for potential differences in HRQL according to the indication for esophagectomy.

In the present study, we have extended the evaluation of quality of life after esophagectomy and gastric pull-up to 10 years and beyond. At a median of 12 and a maximum follow-up of 19 years, we found that the SF-36 scores were greater than the national norms for all but 1 category. The lowest scoring category was physical function, and for this category, our patients were at the national norm. Thus, similar to other reports with shorter follow-up periods, physical function was the last category to recover, lagging behind the other categories even a decade after surgery. However, at a median follow-up of 12 years after esophagectomy and gastric pull-up, physical function had reached a level similar to that of the general population. It is likely that by a decade or more after their cancer 


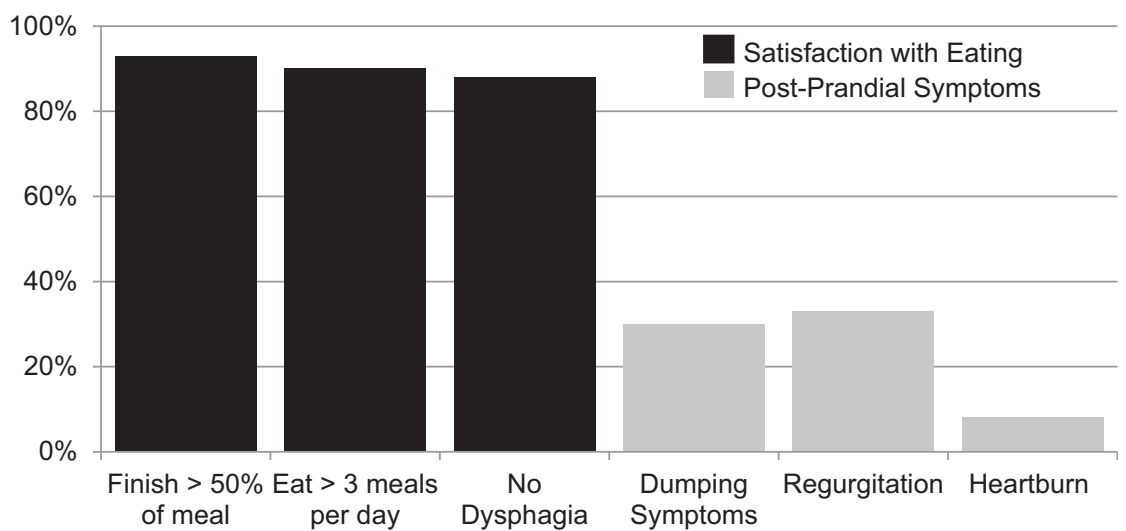

FIGURE 1. Alimentary satisfaction and postprandial symptoms. Alimentary satisfaction was excellent with $>93 \%$ of patients finishing $\geq 50 \%$ of a typical meal, $90 \%$ able to eat $\geq 3$ meals daily, and $88 \%$ of patients reporting no dysphagia. Postprandial symptoms of dumping and diarrhea $\geq 3$ times daily occurred in $33 \%$ of patients and heartburn in $8 \%$ of patients.

treatment, our patients knew they were cured, and this could have influenced their assessment of their quality of life, in particular, for categories such as emotional well-being and social function. The mean cumulative GIQLI score was 88.7 , similar to the score reported for patients with stable gastrointestinal disease (score, 90$)^{9}$; but significantly less than the scores reported for patients who had undergone laparoscopic Nissen fundoplication for gastroesophageal reflux disease (score, 110), ${ }^{10}$ laparoscopic Heller myotomy for achalasia (score, 119), ${ }^{11}$ or normal volunteers (score, 125). ${ }^{9}$ We could not identify any pre- or perioperative variable (eg, age, gender, stage, neoadjuvant chemotherapy, or BMI) that predictably influenced the quality of life (data not shown).

We also evaluated alimentary satisfaction and gastrointestinal symptoms $\geq 10$ years after esophagectomy with gastric pull-up. Our patient population was very uniform in that all had their grafts placed in the posterior mediastinum, all had undergone cervical esophagogastric anastomosis, and all but 1 had received a pyloroplasty. Only $12 \%$ of the patients had dysphagia when evaluated as any or none. Nearly all patients ate $\geq 3$ meals a day and finished $\geq 50 \%$ of a normal-size plate of food with each meal. The mean rating for alimentary comfort was

TABLE 3. Alimentary satisfaction scores (total score, 10)

\begin{tabular}{lc}
\hline \multicolumn{1}{c}{ Score } & Patients $(\mathbf{n}=\mathbf{4 0})$ \\
\hline 10 & $15(37.5)$ \\
9.5 & $3(7.5)$ \\
9 & $3(7.5)$ \\
8.5 & $5(12.5)$ \\
8 & $7(17.5)$ \\
7.5 & $2(5)$ \\
6 & $2(5)$ \\
5.5 & $2(5)$ \\
Unable to answer & $1(2.5)$ \\
\hline
\end{tabular}

Data presented as $\mathrm{n}(\%)$.
9 of a possible 10, with the lowest score 5.5, indicating that most patients were very satisfied and comfortable with their esophageal replacement. A common patient response was that it was not like being "normal," but it was very close. Over time, most patients had been able to identify problematic foods (typically ice cream and milkshakes) and avoid them. Patients typically reported feeling weak and nauseous after consuming these foods, followed rapidly by dumping symptoms. However, over the years,

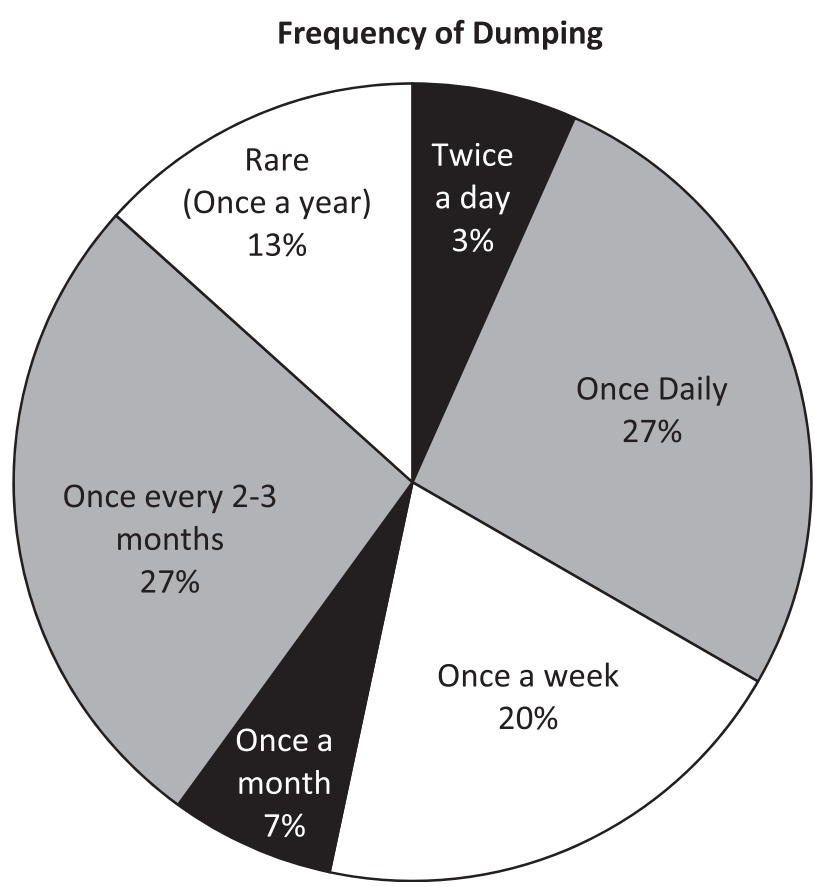

FIGURE 2. Frequency of dumping symptoms. Information on the frequency of dumping was available from 31 patients. Dumping occurred less frequently than once a month in $40 \%$, and no patient reported dumping symptoms with every meal. 
TABLE 4. Preoperative versus postoperative BMI $(n=35)$

\begin{tabular}{lcc}
\hline $\begin{array}{c}\text { BMI range classification } \\
\left(\mathbf{k g} / \mathbf{m}^{\mathbf{2}}\right)\end{array}$ & $\begin{array}{c}\text { Preoperative BMI } \\
\left(\mathbf{k g} / \mathbf{m}^{\mathbf{2}}\right)\end{array}$ & $\begin{array}{c}\text { Current BMI } \\
\left(\mathbf{k g} / \mathbf{m}^{\mathbf{2}}\right)\end{array}$ \\
\hline Underweight $(<18.5)$ & 0 & $2(6)$ \\
Normal range $(18.5$ to $<25)$ & $5(14)$ & $19(54)$ \\
Overweight $(25$ to $<30)$ & $19(54)$ & $10(29)$ \\
Obese class I $(30-35)$ & $10(29)$ & $3(9)$ \\
Obese class II $(>35)$ & $1(3)$ & $1(3)$ \\
\hline$B M I$, Body
\end{tabular}

$B M I$, Body mass index.

most patients had found a home remedy that helped treat the dumping symptoms when they occurred.

After esophagectomy, all patients had lost weight, and the median weight loss at $\geq 10$ years of follow up was 26 lbs. Preoperatively, most patients had been overweight $(54 \%)$, and $32 \%$ were obese. However, $\geq 10$ years after esophagectomy, $32 \%$ of patients were overweight and only $11 \%$ obese. With the exception of 2 patients who were underweight, most patients were maintaining a healthier weight at long-term follow-up. Thus, most of the weight loss with esophagectomy was healthy, and maintaining adequate nutrition was not problematic or difficult.

The most common gastrointestinal symptoms $\geq 10$ years after esophagectomy were early satiety, dumping, frequent diarrhea, and regurgitation. Most patients required daily acid suppression therapy. The return of gastric acid production has been shown to occur as early as 6 months after esophagectomy with gastric tube creation and to increase over time. ${ }^{15}$ Chronic respiratory symptoms potentially related to reflux events such as cough and breathing difficulty were present in $>25 \%$, and aspiration events requiring hospitalization occurred in $15 \%$ of the patients. Regurgitation and aspiration will remain an issue after esophagectomy, because the lower esophageal sphincter has been removed, and the esophagogastric anastomosis is

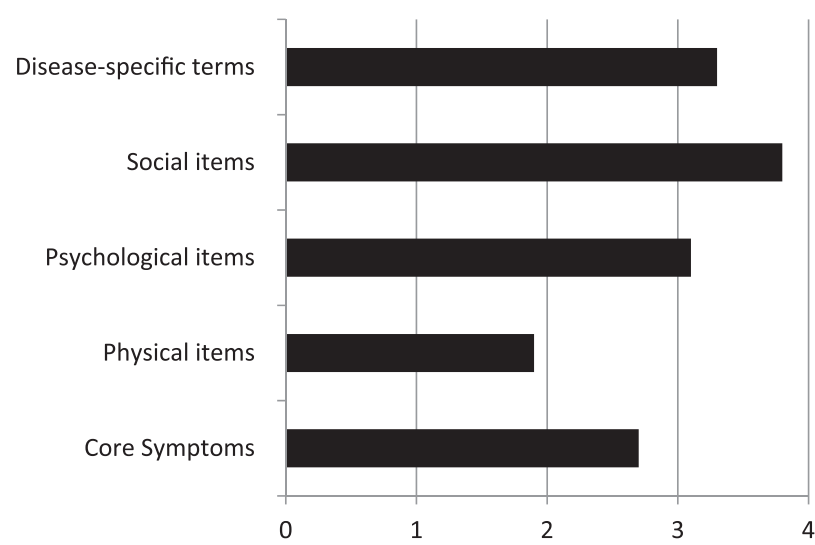

FIGURE 3. Gastrointestinal Quality of Life Index (GIQLI) results. The GIQLI is a validated set of 36 questions in 5 domains, with a maximum score of 4 in each domain. The median GIQLI score for all 5 domains was 2.9 .

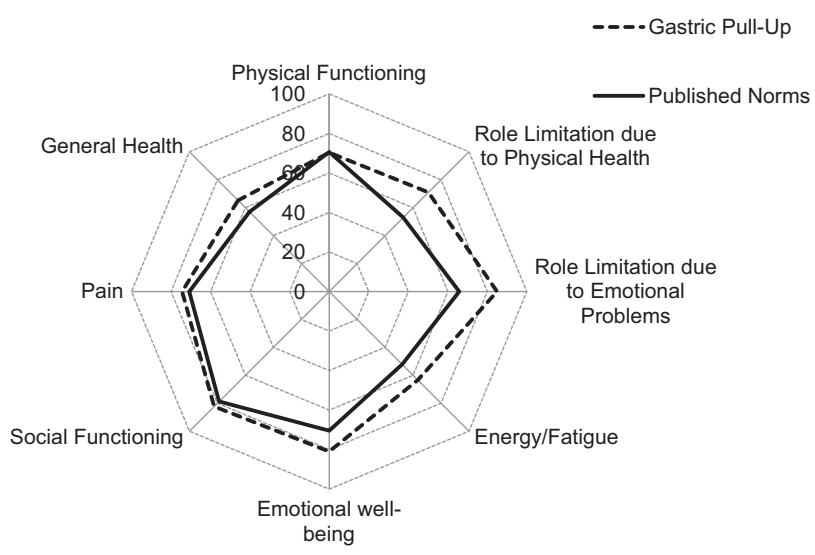

FIGURE 4. The RAND Medical Outcomes Study, short-form, 36-item quality of life scores for the 7 subdomains are shown. Patients $\geq 10$ years after esophagectomy with gastric pull-up were compared with the population mean for each of the domains. The patients had scores greater than the mean in all categories, except for physical function, for which they were at the mean.

typically within 5 to $6 \mathrm{~cm}$ of the larynx. If patients are not diligent about going to bed 2 to 3 hours after their last meal and sleeping with the head of the bed elevated, significant and potentially fatal aspiration can occur. Even occasional aspiration events can ultimately lead to chronic pulmonary damage.

One of the challenges with assessing alimentary satisfaction and gastrointestinal symptoms after esophagectomy is that no validated questionnaires or indexes have been available that apply specifically to these patients. Satisfaction, to a large degree, depends on the expectations of the patient, and these will, in part, be shaped by their personality and lifestyle. Most patients described the first few years after esophagectomy as being very difficult, but gradually they adjusted and, eventually, the eating, digesting, and sleeping became easier. Now, $>10$ years postoperatively, most of the patients appeared to have "mastered" life after esophagectomy. They have exited the learning curve and, with the help of their families and support groups, have adjusted their lifestyles and expectations to function well. They have realized that overeating leads to chest discomfort and dyspnea, and so they have learned when to stop. This has been reflected in that most patients only finished about $50 \%$ of a normal-size plate of food at any one time. The patients who failed to adjust their expectations or to make lifestyle modifications were typically the ones that reported chronic problems with regurgitation and aspiration events and were less satisfied.

The limitations of the present study included the lack of serial follow-up data for the individual patients over time that might have allowed additional insights into the evolution of quality of life, gastrointestinal symptoms, and alimentary satisfaction after esophagectomy and gastric pull-up. Furthermore, we did not have objective follow-up 
data from a barium swallow test, a standardized test meal, or, in most, even endoscopy. Tests such as these could add additional information and insight into graft function and reflux injury to the residual cervical esophagus and should be the focus of future studies. Finally, this small group of patients was an elite group in that they had outlived $83 \%$ of their peers who had undergone esophagectomy during the same period. As such, these patients might not be representative of all patients after esophagectomy.

In conclusion, the quality of life in patients who survive $\geq 10$ years after esophagectomy and gastric pull-up was excellent, matching or exceeding population normal values. The overwhelming majority of our patients were satisfied with their alimentary function, ate $\geq 3$ meals daily, and had a BMI in the normal or overweight range. Furthermore, gastrointestinal symptoms were typically manageable, although troublesome dumping with meals, diarrhea, or regurgitation occurred in one third of the patients. Our findings have shown that pessimism regarding the longterm ability to enjoy a meal and live with a good quality of life after esophagectomy is unwarranted.

\section{References}

1. American Cancer Society. Cancer Facts and Figures 2013. Atlanta: American Cancer Society; 2013.

2. Tatematsu N, Hasegawa S, Tanaka E, Sakai Y, Tsuboyama T. Impact of oesophagectomy on physical fitness and health-related quality of life in patients with oesophageal cancer. Eur J Cancer Care. 2013;22:308-13.

3. Nafteaux P, Durnez J, Moons J, Coosemans W, Decker G, Lerut T, et al. Assessing the relationship between health-related quality of life and postoperative length of hospital stay after oesophagectomy for cancer of the oesophagus and the gastrooesophageal junction. Eur J Cardiothorac Surg. 2013;44:525-33.

4. Derogar M, Lagergren P. Health-related quality of life among 5-year survivors of esophageal cancer surgery: a prospective population based study. J Clin Oncol. 2012;30:413-8.

5. Donohoe C, McGillycuddy E, Reynolds J. Long-term health-related quality of life for disease-free esophageal cancer patients. World J Surg. 2011;35: 1853-60.

6. Scarpa M, Valente S, Alfieri R, Cagol M, Diamantis G, Ancona E, et al. Systematic review of health-related quality of life after esophagectomy for esophageal cancer. World J Gastroenterol. 2011;17:4660-74.

7. Briel J, Tamhankar AP, Hagen JA, DeMeester SR, Johansson J, Choustoulakis E, et al. Prevalence and risk factors for ischemia, leak, and stricture of esophageal anastomosis: gastric pull-up versus colon interposition. J Am Coll Surg. 2004; 198:536-42.

8. Epasch E, Williams J, Dauphinee S, Ure B, Schmulling C, Neugebauer E, et al. Gastrointestinal Quality of Life Index: development, validation and application of a new instrument. Br J Surg. 1995;82:216-22.

9. Ware J, Sherbourne C. The MOS 36-item short-form health survey (SF-36): I. Conceptual framework and item selection. Med Care. 1992;30:473-83.

10. Hauters P, Sorrentino J, Papillon M, Johanet H, Janer R, Auvray S, et al. [Assessment of quality of life after antireflux surgery]. Ann Chir. 2000;125: 948-53.

11. Decker G, Borie F, Bouamrirene D, Veyrac M, Guillon F, Fingerhut A, et al. Gastrointestinal quality of life before and after laparoscopic Heller myotomy with partial posterior fundoplication. Ann Surg. 2002;236:750-8.

12. Sanghera S, Nurkin S, Demmy T. Quality of life after an esophagectomy. Surg Clin North Am. 2012;92:1315-35.

13. Rutegard M, Lagergren P, Rouvelas I, Mason R, Lagergren J. Surgical complications and long-term survival after esophagectomy for cancer in a nationwide Swedish cohort study. Eur J Surg Oncol. 2012;38:555-61.

14. Schneider L, Hartwig W, Aulmann S, Lenzen C, Strobel O, Fritz S, et al. Quality of life after emergency vs. elective esophagectomy with cervical reconstruction. Scand J Surg. 2010;99:3-8.

15. Gutschow C, Collard JM, Romagnoli R, Salizzoni M, Holscher A. Denervated stomach as an esophageal substitute recovers intraluminal acidity with time. Ann Surg. 2001;233:509-14. 